\title{
A Roller Bearing Fault Diagnosis Method Based on LCD Energy Entropy and ACROA-SVM
}

\author{
HungLinh Ao, ${ }^{1,2,3}$ Junsheng Cheng, ${ }^{1,2}$ Kenli Li, ${ }^{4}$ and Tung Khac Truong ${ }^{4,5}$ \\ ${ }^{1}$ State Key Laboratory of Advanced Design and Manufacturing for Vehicle Body, Hunan University, Changsha 410082, China \\ ${ }^{2}$ College of Mechanical and Automotive Engineering, Hunan University, Changsha 410082, China \\ ${ }^{3}$ Faculty of Mechanical Engineering, Industrial University of Ho Chi Minh City, Ho Chi Minh 70550, Vietnam \\ ${ }^{4}$ College of Information Science and Engineering, Hunan University, National Supercomputing Centre in Changsha, \\ Changsha 410082, China \\ ${ }^{5}$ Faculty of Information Technology, Industrial University of Ho Chi Minh City, Ho Chi Minh 70550, Vietnam
}

Correspondence should be addressed to HungLinh Ao; aohunglinh09@yahoo.com.vn

Received 11 April 2013; Accepted 24 June 2013; Published 24 February 2014

Academic Editor: Valder Steffen

Copyright (c) 2014 HungLinh Ao et al. This is an open access article distributed under the Creative Commons Attribution License, which permits unrestricted use, distribution, and reproduction in any medium, provided the original work is properly cited.

\begin{abstract}
This study investigates a novel method for roller bearing fault diagnosis based on local characteristic-scale decomposition (LCD) energy entropy, together with a support vector machine designed using an Artificial Chemical Reaction Optimisation Algorithm, referred to as an ACROA-SVM. First, the original acceleration vibration signals are decomposed into intrinsic scale components (ISCs). Second, the concept of LCD energy entropy is introduced. Third, the energy features extracted from a number of ISCs that contain the most dominant fault information serve as input vectors for the support vector machine classifier. Finally, the ACROASVM classifier is proposed to recognize the faulty roller bearing pattern. The analysis of roller bearing signals with inner-race and outer-race faults shows that the diagnostic approach based on the ACROA-SVM and using LCD to extract the energy levels of the various frequency bands as features can identify roller bearing fault patterns accurately and effectively. The proposed method is superior to approaches based on Empirical Mode Decomposition method and requires less time.
\end{abstract}

\section{Introduction}

Roller bearings are important and frequently encountered components in rotating machines, which are found in widespread industrial applications. Roller bearing fault diagnosis is therefore meaningful. Fault diagnosis includes two aspects: feature extraction and pattern recognition. When a fault occurs in a roller bearing, it is very difficult to extract the fault characteristic information from the nonstationary vibration signals [1,2].

The traditional diagnosis techniques extract the fault characteristic information from the waveforms of the vibration signals in either the time domain or the frequency domain. Then, criterion functions are constructed to identify the condition of the roller bearing. However, it is very difficult to accurately evaluate the condition of a roller bearing through an analysis in the time or frequency domain only $[2,3]$.
The Empirical Mode Decomposition (EMD) method of feature extraction is based on the local characteristic time scale of the signal and can adaptively decompose a complicated multicomponent signal into a sum of intrinsic mode functions (IMFs) whose instantaneous frequencies have physical significance $[4,5]$. By applying an envelope analysis to each IMF component, the characteristic information of original signal can be extracted more accurately and effectively. In addition, the frequency components involved in each IMF are related not only to the sampling frequency but to changes in the signal itself; that is, EMD can be regarded as a self-adaptive filter whose bandwidth and central frequency change with the signal itself. Therefore, EMD is a self-adaptive signal processing method that can be applied to nonlinear and nonstationary processes [6]. However, amplitude and frequency information is lost because of the cubic spline and the Hilbert transform used in the EMD [7]. Rilling 
and Flandrin asserted that two tones can be separated using EMD, and numerical experiments supported their claims [8]. Wu and Huang [9] found that two components whose frequencies lie within an octave cannot be separated by EMD. However, there are currently no rules or guidelines for deciding when two separate components can be separated using EMD. Furthermore, the end effect [10], mode mixing [11], overshoot and undershoot [12], negative frequenciesinstantaneous frequency [13], and a lack of a theoretical foundation [14] are all current drawbacks of EMD.

Recently, Cheng et al. developed a new signal analysis method, the local characteristic-scale decomposition (LCD), that defines intrinsic scale components, and, with the physical instantaneous frequency, this method can decompose a complicated signal into several intrinsic scale components (ISC) [15]. By analysing each resulting ISC, which involves the local characteristic of the signal, the characteristic information of the original signal can be extracted more accuracy and effectively. The LCD method is superior to the HilbertHuang Transform method in reducing the end effect and the iteration time and in the accuracy of the instantaneous characteristic.

Pattern recognition is the other aspect of roller bearing fault diagnosis. Traditional statistical pattern recognition methods and Artificial Neural Network (ANN) classifiers assume that sufficient samples are available, which is not always true in practice [16]. The Support Vector Machine (SVM) is a powerful machine learning method, based on statistical learning theory and the structural risk minimization principle that has been successfully applied in classification and regression problems [17]. SVMs not only can solve the problems of overfitting, local optimal solutions, and slow convergence rates that exist in ANNs, but they also have an excellent generalization capability in situations where there are a small number of samples. Furthermore, SVMs can solve nonlinear, high-dimensional pattern recognition problems with a limited number of samples and represent nonlinear relationships between the input and the output [18]. SVMs have been found to be remarkably effective in many practical applications. This method is widely used in areas such as pattern recognition [19], time-series forecasting [20], diagnostics [21-25], robotics [26], signal processing [25, 27], speech and word recognition [28], machine vision [29], and financial forecasting [30]. In SVMs, the kernel parameters have an influence on the generalization performance, and the regularization constant $C$ determines the trade-off between minimizing the training error and minimizing the model complexity. The parameters of the kernel function implicitly define the nonlinear mapping from the input space to the high-dimensional feature space [31]. The performance of the SVM will be degraded if these parameters are not properly chosen. There are several methods for choosing the parameters of the SVM such as trial-and-error procedures [32], the grid algorithm [33], the cross-validation method [34], the generalization error estimation method [35], and the gradient descent method [36]. These methods have several drawbacks; for example, both the grid method and the crossvalidation method require long and complicated calculations [33].
In recent years, heuristic algorithms have been developed and are widely used. These algorithms use simple approaches found in heuristic optimisation algorithms. Some heuristic algorithms that have been used to optimise SVM parameters are Ant Colony Optimization (ACO) [37], Particle Swarm Optimization (PSO) [38-40], the Genetic Algorithm (GA) [41], and the Bee Colony Algorithm (BCA) [42]. The Artificial Chemical Reaction Optimisation Algorithm (ACROA), which was introduced by Bilal Alatas, is a novel computational method that was inspired by chemical reactions [43]. The ACROA has been applied successfully to optimisation problems and the mining of classification rules [44]. Compared with the GA, the ACROA can reach a global optimum in a very short time, and the classification result is the same. The ACROA is adapted to the solution procedure to an optimisation problem. This algorithm is based on the second law of thermodynamics; that is, a system tends toward the highest entropy and the lowest enthalpy [45]. In the ACROA, the enthalpy or potential energy and the entropy can be used as the objective functions for the minimisation and maximisation problems, respectively, for the optimisation problem of interest. The ACROA is robust, and thus we chose the ACROA to solve our problem. In this study, the ACROA is applied to optimise the SVM parameters.

In this paper, LCD is applied to diagnose the roller bearing faults. First, the original acceleration vibration signal is decomposed using LCD and the ISC components are obtained, and then the concept of LCD energy entropy is introduced, which can reflect the actual condition and the fault pattern of the roller bearing. The LCD energy entropies of different vibration signals illustrate that the energy of the vibration signal in different frequency bands will change when a bearing fault occurs. To identify the condition of the roller bearing further, the ACROA-SVM serves as a classifier, and the extracted energy features of the stationary ISCs are taken as classifier input vectors so that a faulty bearing can be distinguished from a normal bearing. To verify the superiority of the LCD method, it is compared with the EMD method. As in the LCD method, the original signal is decomposed with EMD, and then the energy features are extracted. These resulting features are also used as input vectors to the ACROA-SVM to identify the condition of a roller bearing. The experimental results show that the diagnostic approach of the ACROA-SVM based on LCD energy entropy has better identification accuracy than EMD and is faster.

The remainder of this paper is organised as follows. Section 2 discusses the LCD method. In Section 3, the concept of LCD energy entropy is proposed, and the LCD energy entropies of different vibration signals are calculated to illustrate that the energy of an acceleration signal in different frequency bands changes when a roller bearing fault occurs. Section 4 explains the ACROA and the parameter optimisation of an SVM based on the ACROA. In Section 5, the fault diagnosis method based on LCD and the ACROA$\mathrm{SVM}$ is given, in which the energy features extracted from a number of ISCs are used as input vectors to the ACROASVM. In Section 6, the fault diagnosis method is used to diagnose the condition of actual roller bearings and is 


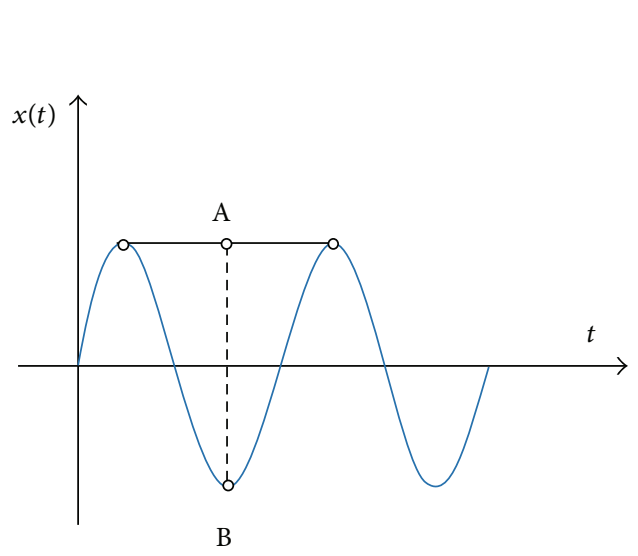

(a) A sine signal

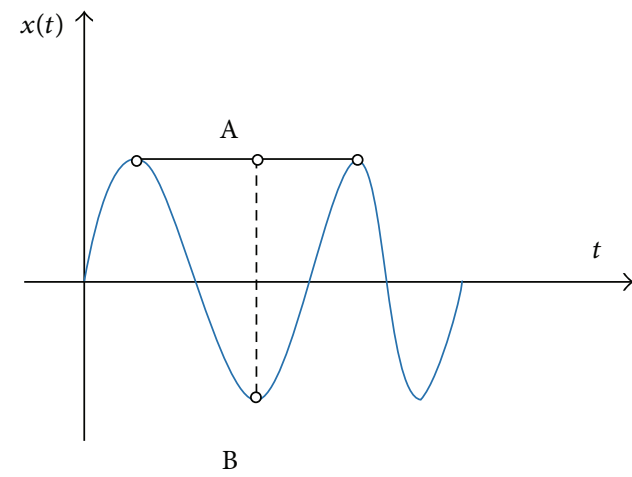

(c) A FM signal

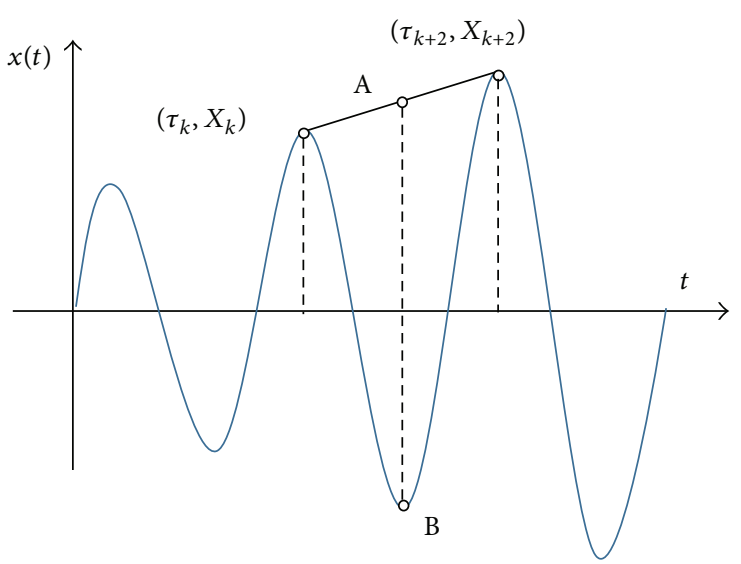

(b) An AM signal

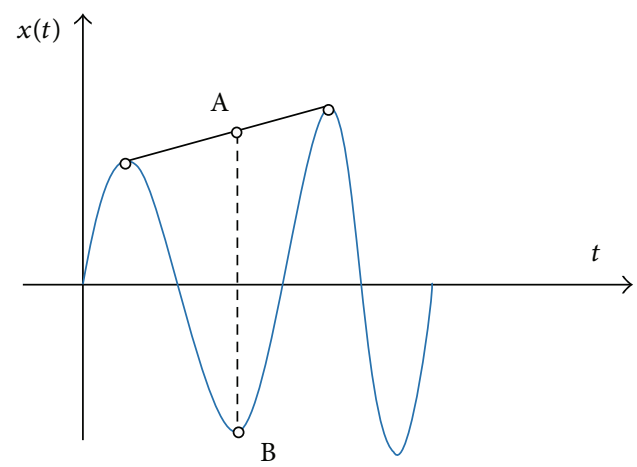

(d) An AM-FM signal

FIGURE 1: Four types of typical signal owning instantaneous frequency with physical meaning.

compared with the EMD method. The conclusions drawn from this research are given in Section 7.

\section{LCD Method}

Figure 1 shows four types of signals having an instantaneous frequency with physical significance. In Figure 1, point A is the value of the line connecting two adjacent peaks at the time where the minimum occurs between the two maximums, and point $B$ is the minimum point. The LCD method is developed from the simple assumptions that any complicated signal consists of several ISCs and any two ISCs are independent of each other. In this way, each signal can be decomposed into a number of ISCs, each of which must satisfy the following definition [15].

(I) In the entire data set, all the local maxima are positive, all the local minima are negative, and the signal is monotonic between any two adjacent extreme points.

(II) Among the data, let all the maximal points be denoted as $\left(\tau_{k}, X_{k}\right), k=1,2, \ldots, M$, where $M$ is the number of maximal points.
The line formed by any two adjacent extreme points, $l_{k}$, at the $\tau_{k+1}$ as $A_{k+1}$, is specified as follows:

$$
l_{k}=\frac{\left(X_{k+2}-X_{k}\right)\left(t-\tau_{k}\right)}{\left(\tau_{k+2}-\tau_{k}\right)}+X_{k}
$$

Then, the relation

$$
a A_{k+1}+(1-a) X_{k+1}=0, \quad a \in(0,1),
$$

should be true, where

$$
A_{k+1}=\frac{\left(\tau_{k+1}-\tau_{k}\right)}{\left(\tau_{k+2}-\tau_{k}\right)}\left(X_{k+2}-X_{k}\right)+X_{k}
$$

Generally, $a=0.5$ when $A_{k+1}=-X_{k+1}$.

Based on the definition of the ISC component, a realvalued signal, $x(t)(t>0)$, can be decomposed into a number of ISCs using the LCD method in the following way.

(1) Let $\left(\tau_{k}, X_{k}\right)(k=1,2, \ldots, M)$ denote the extrema of the signal $x(t)$, and let $A_{k}(k=2,3, \ldots, M-1)$ be calculated as in (3). Then, the values $L_{k}(k=2,3, \ldots, M-1)$ can be calculated as follows:

$$
L_{k}=\frac{A_{k}+X_{k}}{2}, \quad k=2,3, \ldots, M-1 .
$$

Note that the values of $A_{k}$ and $L_{k}$ are for $k$ from 2 to $M-1$. Hence, we must extend the boundaries of the data, which can 
be achieved in many different ways, such as the methods used in EMD [10, 46].

By extension, the two end extrema $\left(\tau_{0}, X_{0}\right)$ and $\left(\tau_{M+1}, X_{M+1}\right)$ can be obtained. According to (3) and (4), we can obtain $L_{1}$ and $L_{M}$. Otherwise, we can extend the sequence $L_{k}$ directly from the known values.

(2) Connect all the $L_{k}$ with a cubic spline to form the base line, denoted as $B_{1}(t)$. Theoretically, the difference between the original data and the base line, $B_{1}(t)$, is the first ISC, $h_{1}(t)$; that is,

$$
h_{1}(t)=x(t)-B_{1}(t) .
$$

If $h_{1}(t)$ meets conditions (I) and (II), then it is an ISC component, and $h_{1}(t)$ is chosen as the first ISC.

(3) Or see the $h_{1}(t)$ as the original data, and repeat the above step, defining

$$
h_{11}(t)=h_{1}(t)-I_{11}(t) \text {. }
$$

If $h_{11}(t)$ does not satisfy conditions (I) and (II), repeat this step $k$ times until $h_{1 k}(t)$ satisfies the ISC conditions; then denote $h_{1 k}(t)$ as the first ISC, $I_{1}(t)$.

(4) Separate $I_{1}(t)$ from the initial data, and define the residue as $u_{1}(t)$ :

$$
u_{1}(t)=x(t)-I_{1}(t)
$$

(5) Next, add $u_{1}(t)$ to the original data, and repeat steps $(1)-(4)$. Similarly, we obtain $I_{2}(t), \ldots, I_{n}(t)$ until the residue $u_{n}(t)$ is either a monotonic or a constant function. Then, $x(t)$ is decomposed into $n$ ISCs and a residue $u_{n}(t)$; that is,

$$
x(t)=\sum_{i=1}^{n} I_{i}(t)+u_{n}(t) .
$$

Similar to the Cauchy convergence test, the standard deviation (SD) is defined as

$$
\mathrm{SD}=\sum_{t=0}^{T}\left[\frac{\left|h_{i k}(t)-h_{i(k-1)}(t)\right|^{2}}{h_{i(k-1)}^{2}(t)}\right]
$$

where $T$ is the length of time. The sifting process is stopped when SD is less than a chosen value. Generally, a value of SD less than 0.3 is ideal for an ISC.

Figure 2 shows a multicomponent, modulated signal that is defined by the following function:

$$
\begin{aligned}
x(t)= & 2 \sin \left(2 \pi f_{1} t\right)+4 \sin \left(2 \pi f_{2} t\right) \sin \left(2 \pi f_{3} t\right) \\
& +\sin \left(2 \pi f_{4} t\right),
\end{aligned}
$$

where $f_{1}=15, f_{2}=10, f_{3}=0.1$, and $f_{4}=5$.

The decomposed results in Figure 3 show that the LCD method is superior to the EMD method with the same steps of decomposition. Intuitively, in contrast with IMFs, ISCs provide more information on the modulation characteristics.

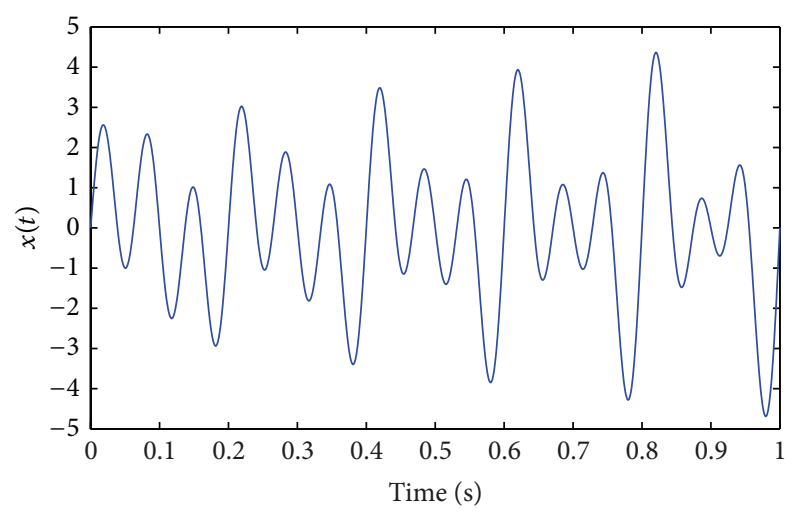

FIGURE 2: The multicomponent modulated signal.

TABLE 1: The LCD energy entropies of the vibration signals of the roller bearing with different faults.

\begin{tabular}{lcc}
\hline Normal & Outer-race fault & Inner-race fault \\
\hline 1.1504 & 1.0887 & 0.8333 \\
\hline
\end{tabular}

\section{LCD Energy Entropy}

The vibration signal from a faulty roller bearing reflects the corresponding resonant frequency components, and its energy changes with the frequency distribution. Therefore, in this study, the LCD energy entropy is proposed to capture this change.

It is assumed that the vibration signal of a faulty roller bearing $x(t)$ has been decomposed with LCD into $n$ ISCs and a residue $u_{n}(t)$, where the energies of the $n$ ISCs are $E_{1}, E_{2}, \ldots, E_{n}$. The sum of the energies of the $n$ ISCs should be equal to the total energy of the original signal when the residue $u_{n}(t)$ is ignored. Because the ISCs $I_{1}(t), I_{2}(t), \ldots, I_{n}(t)$ include various frequency components, $E=\left\{E_{1}, E_{2}, \ldots, E_{n}\right\}$ forms an energy distribution in the frequency domain of the roller bearing vibration signal. The corresponding LCD energy entropy is defined as

$$
H_{\mathrm{EN}}=-\sum_{i=1}^{n} q_{i} \log q_{i}
$$

where $q_{i}=E_{i} / E$ is the percentage of the energy of $I_{i}(t)$ in the total signal energy $\left(E=\sum_{i=1}^{n} E_{i}\right)$.

Figures 4(a), 4(b), and 4(c) show the three cases of the roller bearing vibration signal: normal, with an outer-race fault and with an inner-race fault, respectively. Table 1 shows that the energy entropy of the vibration signal of a normal roller bearing is greater than that of the others because the energy distribution of this kind of signals in each frequency band is comparatively even and uncertain. For a roller bearing with an outer-race fault, the energy entropy is lower because the energy is distributed mainly in the resonant frequency band and the distribution uncertainty is lower. Moreover, the higher resonant frequency components are produced in the roller bearing with an inner-race fault and the impact is more severe, so the energy entropy in this case would be the least. 


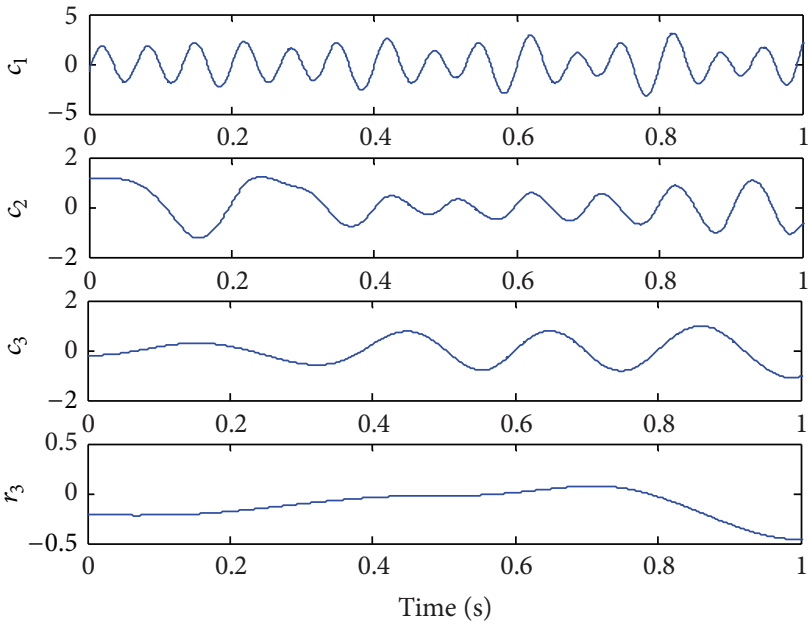

(a)

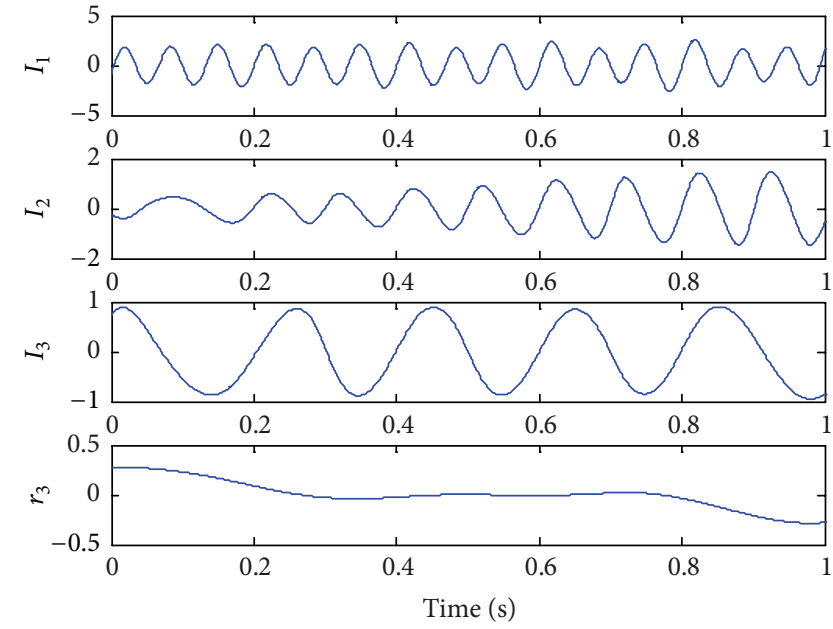

(b)

FIGURE 3: The EMD (a) and LCD (b) decomposed results of the multicomponent modulated signal shown in Figure 2.

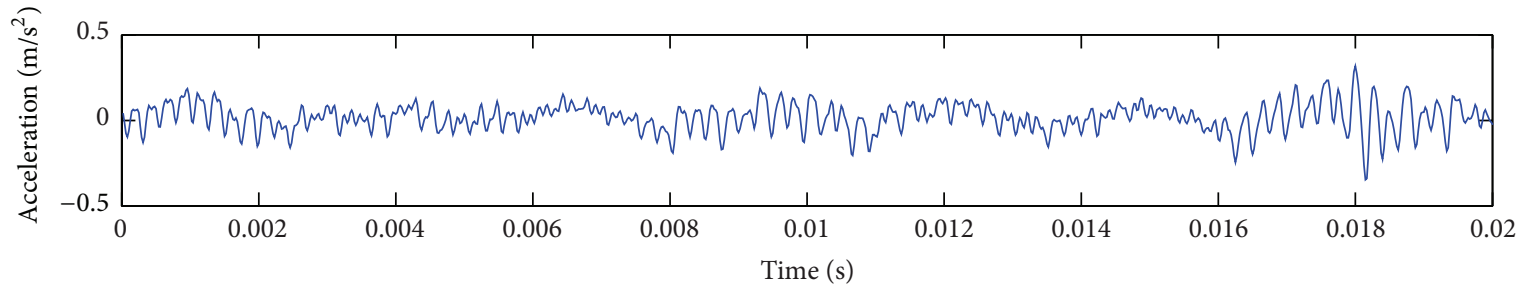

(a)

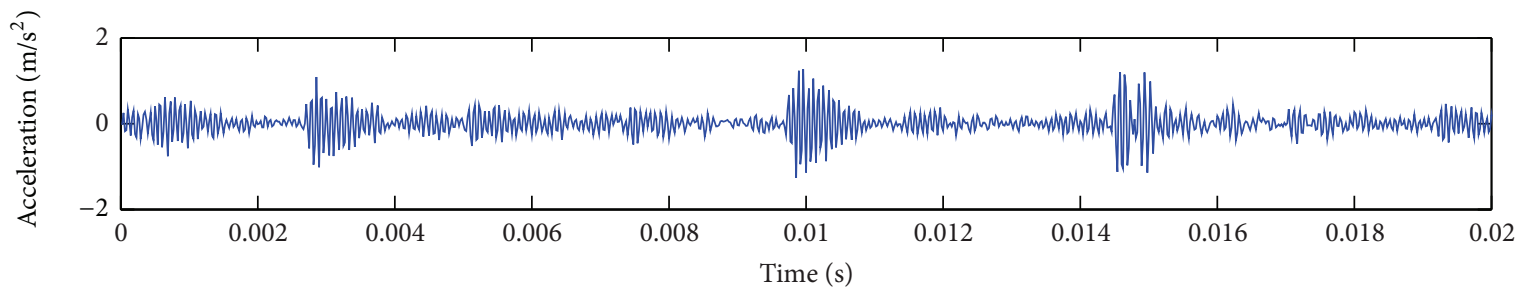

(b)

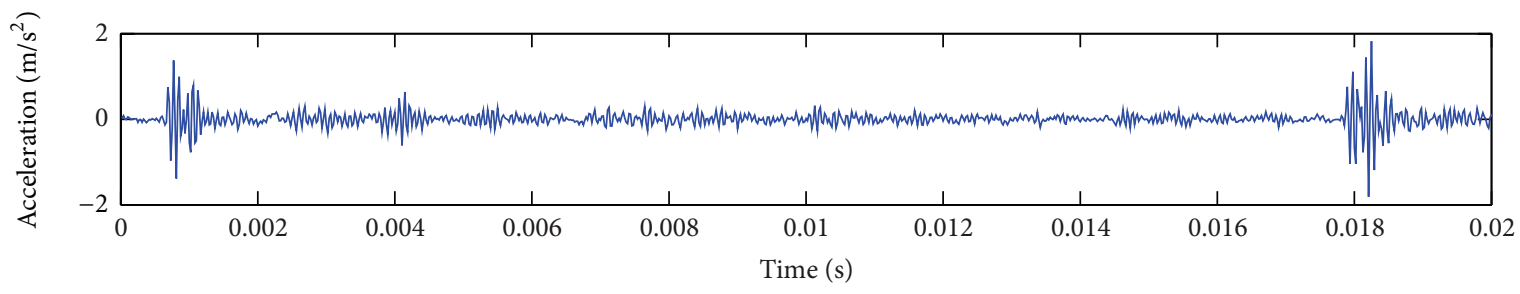

(c)

FIGURE 4: The vibration acceleration signal of the normal roller bearing (a), out-race fault (b), and inner-race fault (c), respectively.

It can be concluded from the preceding analysis that the energy entropy based on LCD can reflect the condition and the fault pattern of the roller bearing. However, for each roller bearing, the LCD energy entropy varies for the same condition. Therefore, it is not sufficient to distinguish the condition and the fault pattern only according to the LCD energy entropy; further analysis is desirable.

\section{ACROA-SVM}

4.1. Artificial Chemical Reaction Optimization Algorithm. The ACROA is an adaptive optimisation technique. The stochastic search algorithm of the ACROA is based on the process of chemical reactions. A chemical reaction is a process that leads to the transformation of one set of chemical substances into 


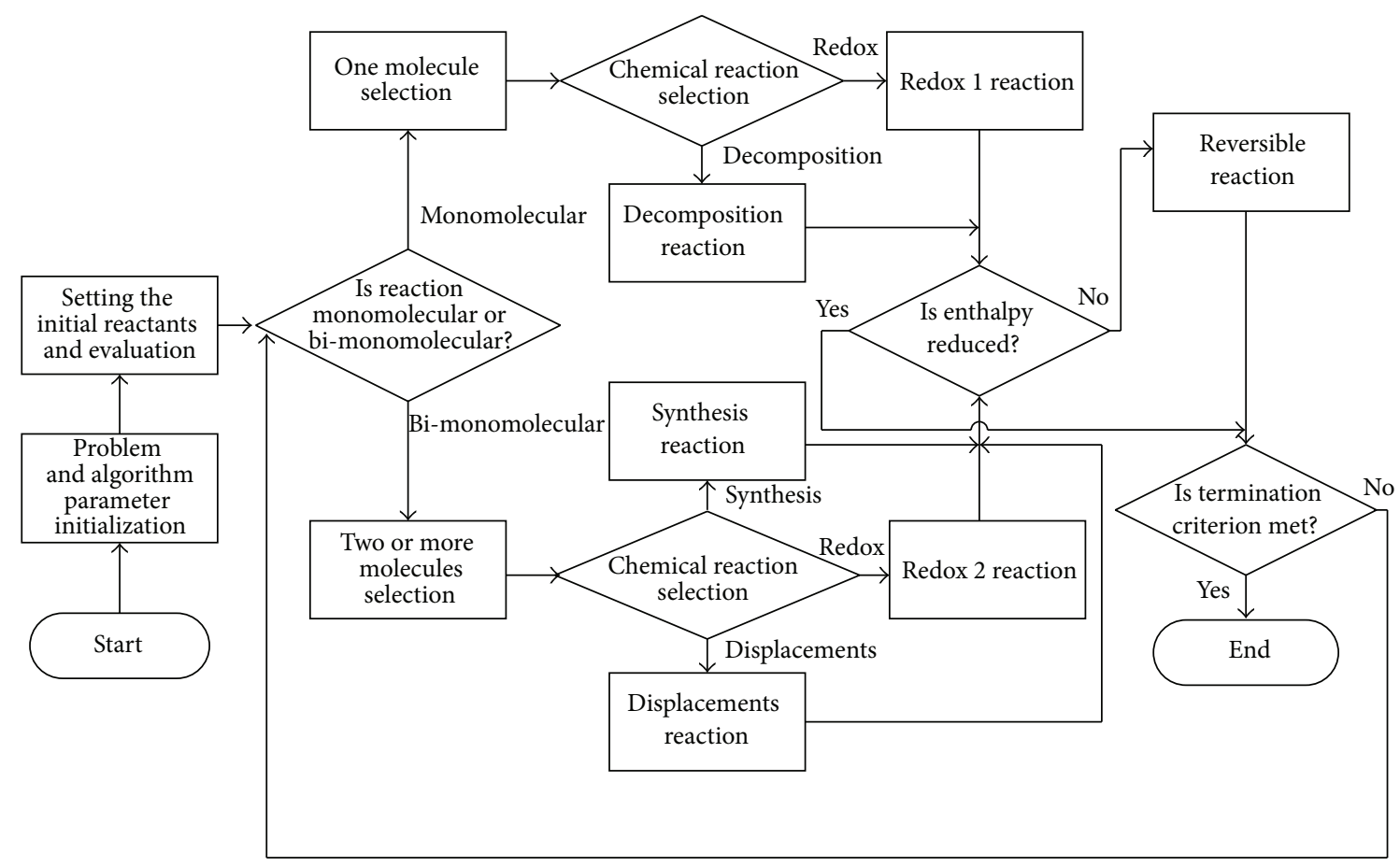

FIgURE 5: Flow chart of ACROA [43].

another. Two key reactions in the ACROA are bimolecular and monomolecular reactions [43]. The principle of the ACROA is presented in the flow chart in Figure 5 and consists of the following five steps [44].

Step 1. Define the problem and the algorithm parameters.

Step 2. Initialize the reactants and evaluate them.

Step 3. Simulate the chemical reactions.

Step 4. Update the reactants.

Step 5. Check the termination criterion.

The optimization problem is specified as follows:

maximize $f(x)$

subject to $x_{j} \in D_{j}=\left[l_{j}, u_{j}\right], \quad j=\{1,2, \ldots, N\}$,

where $f(x)$ is a fitness function, $x=\left(x_{1}, x_{2}, \ldots, x_{N}\right)$ is the vector of decision variables, $N$ is the number of decision variables, and $D_{j}$ is the range of feasible values for decision variable $j$, where $l_{j}$ and $u_{j}$ are the lower and upper bounds of the $j$ th decision variable, respectively. More details about these steps can be found in $[43,44]$.

4.2. Support Vector Machine (SVM). The SVM is developed from the optimal separation plane under linearly separable conditions. The basic idea of the SVM is to map the training samples from the input space into a higher dimensional feature space via a mapping function $\phi$ [37]. Suppose there is a given training sample set $G=\left\{\left(x_{i}, y_{i}\right), i=1,2, \ldots, l\right\}$, where each sample $x_{i} \in R^{d}$ belongs to a class determined by $y \in$ $\{+1,-1\}$. When the training data are not linearly separable in the feature space, the target function can be expressed as follows [17]:

$$
\begin{array}{ll}
\min & \phi(\omega)=\frac{1}{2}\langle\omega \cdot \omega\rangle+C \sum_{i=1}^{l} \xi_{i} \\
\text { s.t } & y_{i}\left(\left\langle\omega \cdot \phi\left(x_{i}\right)\right\rangle+b\right) \geq 1-\xi_{i}, \\
& \xi_{i} \geq 0, \quad i=\{1,2, \ldots, l\},
\end{array}
$$

where $\omega$ is the normal vector of the hyperplane, $C$ is a penalty parameter, $b$ is the bias that is a scalar, $\xi_{i}$ are nonnegative slack variables, and $\phi(x)$ is a mapping function.

By introducing Lagrange multipliers $\alpha_{i} \geq 0$, the optimization problem can be rewritten as follows.

Maximize

$$
L(\omega, b, \alpha)=\sum_{i=1}^{l} \alpha_{i}-\frac{1}{2} \sum_{i, j=1}^{l} \alpha_{i} \alpha_{j} y_{i} y_{j} K\left(x_{i} \cdot x_{j}\right) .
$$

Subject to

$$
\begin{aligned}
& 0 \leq \alpha_{i} \leq C \\
& \sum_{i=1}^{l} \alpha_{i} y_{i}=0 .
\end{aligned}
$$

The decision function can be obtained as follows:

$$
f(x)=\operatorname{sign}\left(\sum_{i=1}^{l} \alpha_{i} y_{i} K\left(x_{i} \cdot x\right)+b\right) .
$$


The most common kernel functions used in SVMs are as follows:

(i) linear kernel

$$
K\left(x, x_{i}\right)=\left\langle x \cdot x_{i}\right\rangle,
$$

(ii) polynomial kernel

$$
K\left(x, x_{i}\right)=\left(\left\langle x \cdot x_{i}\right\rangle+c\right)^{d},
$$

(iii) RBF kernel

$$
K\left(x, x_{i}\right)=\exp \left(-\frac{\left\|x-x_{i}\right\|^{2}}{2 \sigma^{2}}\right),
$$

where $d$ and $\sigma$ are kernel parameters.

In this paper, the radial basis function kernel is used because of its universal application and good performance.

4.3. Optimisation of the SVM Parameters Using the ACROA. SVM parameters have an important effect on the classification accuracy. The parameters of the Gaussian kernel function include a penalty factor $C$ and the standard deviation $\sigma$. The selection of the SVM parameters is very difficult. Generally, $C$ and $\sigma$ are selected according to experience. In this paper, the ACROA is used to optimise the parameters of the SVM. These variables are $C$ and $\sigma$, and the fitness function is the accuracy of the SVM. The fitness of the SVM is defined as follows:

$$
\text { fitness }(x)=\operatorname{accuracy}_{\mathrm{SVM}}(x),
$$

where $x=(C, \sigma)$, and the accuracy of the SVM is defined as

$$
\begin{aligned}
& \text { accuracy }_{\text {SVM }} \\
& =\frac{\text { Number of correct classifications of test samples }}{\text { Total number of samples in test set }} .
\end{aligned}
$$

The flow chart of the ACROA-SVM is shown in Figure 6.

4.4. Experimental Results. To evaluate the performance of the proposed ACROA-SVM method, we used three common benchmark data sets from the UCI benchmark, the Iris, Thyroid, and Seed data sets. The sizes of the training and test sets can be found in Table 2 .

The Iris data set contains 150 instances and four attributes. In this data set, the class attribute is typed and there are three classes: Setosa, Versicolor, and Virginica.

The Thyroid data set is used for the diagnosis of hyperthyroidism or hypothyroidism. This data set contains 215 patterns and 5 attributes, and there are three classes: normal, hyper, and hypo.

The Seed data set was obtained from the high-quality visualization of the internal structure of wheat kernels. The Seed data set contains 210 instances of wheat samples with 7

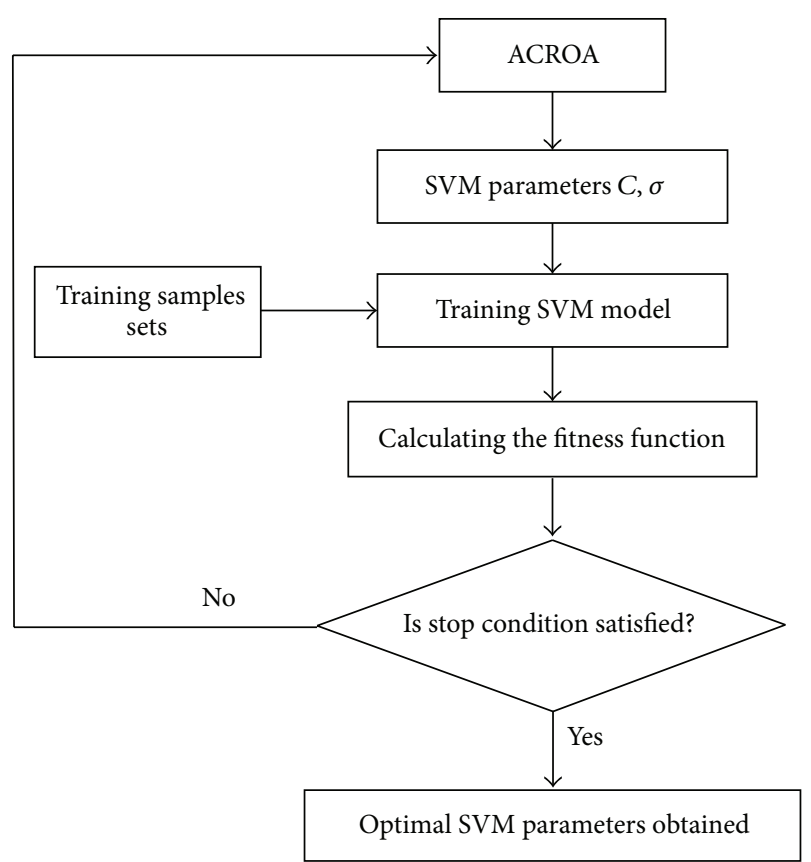

FIgURE 6: Parameter optimization flow chart of SVM based on ACROA.

TABle 2: Properties of the problems.

\begin{tabular}{lccccc}
\hline Name & Data & Train & Test & Input & Class \\
\hline Iris & 150 & 114 & 36 & 4 & 3 \\
Thyroid & 215 & 162 & 53 & 5 & 3 \\
Seed & 210 & 159 & 51 & 7 & 3 \\
\hline
\end{tabular}

inputs, and there are three classes included: Kama, Rosa, and Canadian.

Four methods, the ACROA-SVM, the GA-SVM, the PSOSVM, and the SVM, were used to classify these data sets. For the GA, the generation and population sizes were set to 50 and 20 , respectively. To make a fair comparison, the values of the ACROA were chosen to be the same, for example, iterations $=50$, ReacNum $=20$. For the PSO, the parameters were fixed with the values given in the literature $[39,40]$; that is, $W=$ $0.75, c_{1}=c_{2}=1.5$, the numbers of particles was 20 , and the iteration count was 50. In the SVM method, the values of $C$ and $\sigma$ were chosen by default, and thus the computation time was not calculated. The results in Tables 3,4 , and 5 show that the values of $C$ and $\sigma$ obtained by each method are different. The test error value of the ACROA-SVM method is better than those of the GA-SVM, the PSO-SVM, and the SVM method. Furthermore, the computation time of the proposed ACROA-SVM method is less than those of the GA-SVM and the PSO-SVM methods. The ACROA-SVM method was next applied to a roller bearing fault diagnosis problem.

\section{Roller Bearing Fault Diagnosis Method Based on LCD and ACROA-SVM}

It can be observed from the preceding analysis that the LCD energy entropies of the vibration signals of the roller 
TABLE 3: The identification result of IRIS data set.

\begin{tabular}{|c|c|c|c|c|c|c|}
\hline Method & Training samples & Test samples & $C$ & $\sigma$ & Cost time (s) & Test error (\%) \\
\hline ACROA-SVM1 & 114 & 36 & 224.55 & 4.26 & 83.76 & $\mathbf{0}$ \\
\hline ACROA-SVM2 & 76 & 24 & 240.30 & 4.83 & 43.7128 & $\mathbf{0}$ \\
\hline GA-SVM1 & 114 & 36 & 181.0026 & 1.24 & 543.63 & 0 \\
\hline GA-SVM2 & 76 & 24 & 180.0001 & 1.32 & 231.91 & 0 \\
\hline PSO-SVM1 & 114 & 36 & 185.34 & 1.36 & 426.89 & 0 \\
\hline PSO-SVM2 & 76 & 24 & 179.21 & 1.45 & 247.12 & 0 \\
\hline SVM1 & 114 & 36 & 200 & 1 & I & 0 \\
\hline SVM2 & 76 & 24 & 200 & 1 & l & 4.17 \\
\hline
\end{tabular}

TABLE 4: The identification result of THYROID data set.

\begin{tabular}{|c|c|c|c|c|c|c|}
\hline Method & Training samples & Test samples & $C$ & $\sigma$ & Cost time (s) & Test error (\%) \\
\hline ACROA-SVM1 & 162 & 53 & 214.09 & 4.91 & 187.51 & 7.55 \\
\hline ACROA-SVM2 & 142 & 43 & 226.97 & 1.06 & 91.814 & 4.65 \\
\hline GA-SVM1 & 162 & 53 & 245.62 & 2.98 & 1239.72 & 11.33 \\
\hline GA-SVM2 & 142 & 43 & 224.77 & 2.62 & 407.33 & 9.3 \\
\hline PSO-SVM1 & 162 & 53 & 248.73 & 3.01 & 1189.83 & 11.33 \\
\hline PSO-SVM2 & 142 & 43 & 226.18 & 2.75 & 397.25 & 9.3 \\
\hline SVM1 & 162 & 53 & 200 & 1 & I & 18.87 \\
\hline SVM2 & 142 & 43 & 200 & 1 & I & 18.61 \\
\hline
\end{tabular}

TABLE 5: The identification result of SEED data set.

\begin{tabular}{lcccccc}
\hline Method & Training samples & Test samples & $C$ & $\sigma$ & Cost time (s) & Test error (\%) \\
\hline ACROA-SVM1 & 159 & 51 & 16356.01 & 4.31 & $\mathbf{2 3 8 . 0 6}$ & $\mathbf{0}$ \\
ACROA-SVM2 & 106 & 34 & 186.05 & 4.85 & $\mathbf{1 3 5 . 0 7}$ & 3.92 \\
GA-SVM1 & 159 & 51 & 180.1 & 1.01 & 549.44 & 0 \\
GA-SVM2 & 106 & 34 & 180.28 & 2.27 & 484 & 0 \\
PSO-SVM1 & 159 & 51 & 185.26 & 1.35 & 372.11 & 383.45 \\
PSO-SVM2 & 106 & 34 & 178.23 & 1.89 & 1 & 3.92 \\
SVM1 & 159 & 51 & 200 & 1 & 1 & 0 \\
SVM2 & 106 & 34 & 200 & 1 & 1 \\
\hline
\end{tabular}

bearings with different conditions and fault patterns are obviously different, which shows that the energy of each ISC changes when the roller bearing develops a fault. In this paper, by taking the energy feature of each ISC component as the ACROA-SVM input vector, the condition and the fault pattern of the roller bearing can be identified effectively. The flow chart of the roller bearing fault diagnosis method based on LCD and the ACROA-SVM is shown in Figure 7.

The fault diagnosis method consists of the following seven steps [47].

(1) Collect signals from the roller bearings as samples for the three conditions: normal, outer-race fault, and inner-race fault.

(2) Decompose the original vibration signals into several ISCs, and choose the first $m$ ISCs that include the most dominant fault information to extract the feature.
(3) Calculate the total energy $E_{i}$ of the first $m$ ISCs from

$$
E_{i}=\int_{-\infty}^{+\infty}\left|I_{i}(t)\right|^{2} d t, \quad(i=1,2, \ldots, m) .
$$

(4) Construct the feature vector $T$ with the energy levels as its elements as follows:

$$
T=\left[E_{1}, E_{2}, \ldots, E_{m}\right] .
$$

Considering that the energy is sometimes biggest, $T$ is adjusted by normalizing the feature to simplify the subsequent analysis and processing.

Let

$$
E=\left(\sum_{i=1}^{m}\left|E_{i}\right|^{2}\right)^{1 / 2}
$$




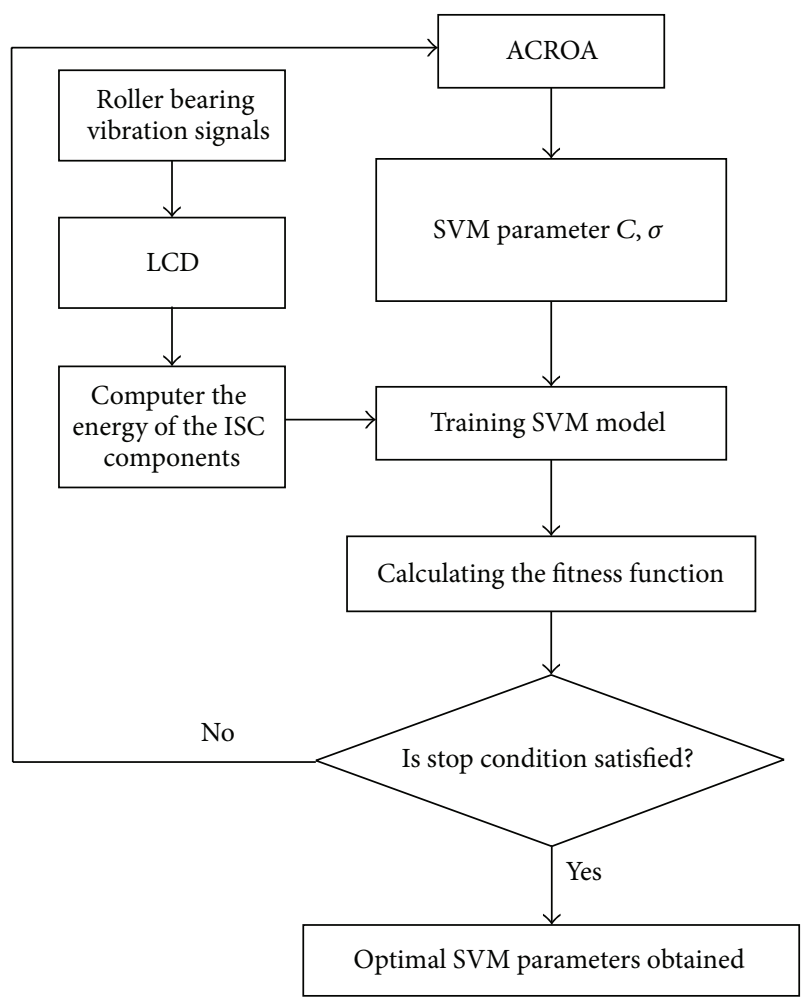

FIGURE 7: The flow chart of the roller bearing fault diagnosis method based on LCD and ACROA-SVM.

Then

$$
T^{\prime}=\left[\frac{E_{1}}{E}, \frac{E_{2}}{E}, \ldots, \frac{E_{m}}{E}\right]
$$

and the vector $T^{\prime}$ is a normalized vector.

(5) Run the ACROA-SVM, where the SVM parameters, namely, $C$ and $\sigma$, are optimized by the ACROA.

(6) Input the vector $T^{\prime}$ into the ACROA-SVM classifier and perform the training. The fitness function is given by (20). The resulting values of $C$ and $\sigma$ are input to the ACROASVM classifier.

(7) For ACROA-SVM1, define the condition of an outerrace fault with the label $y=+1$ and the other conditions with the label $y=-1$. Thus, the outer-race fault can be separated from the other conditions by ACROA-SVM1. Similarly, define the condition of an inner-race fault with the label $y=+1$ and the other conditions with the label $y=-1$ for ACROA-SVM2. Thus, the inner-race fault can be separated from other conditions by ACROA-SVM2. Similarly, the roller fault can be separated from the other conditions by ACROA-SVM3 and so on.

\section{Application}

The test rig is shown in Figure 8 and includes a motor, a coupling, a rotor, and a shaft with two roller bearings. The roller bearings are of the 6311 type. This test rig is popular for testing imbalances, misalignment, and various types of bearing faults. The shaft rotational frequency is

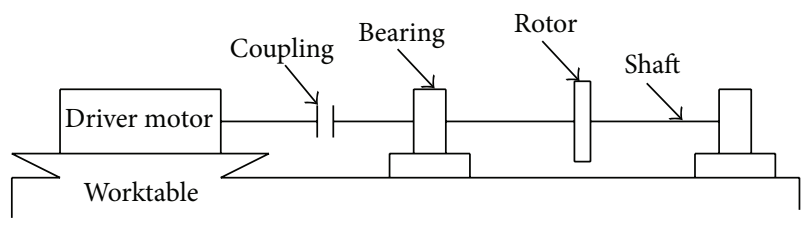

Figure 8: Test rig.

$25 \mathrm{~Hz}$, and the rotor's polar moment of inertia is $0.03 \mathrm{~kg} \cdot \mathrm{m}^{2}$. After the impulse excitation experiment to the roller bearing, the first three resonance frequencies of the roller bearing are determined as $420 \mathrm{~Hz}, 732 \mathrm{~Hz}$, and $1016 \mathrm{~Hz}$, respectively. According to Nyquist sampling theorem, sampling frequency $f_{s}$ is chosen as follows [48]:

$$
f_{s} \geq 2 f_{\max }
$$

where $f_{s}$ is the sampling frequency and $f_{\max }$ is the highest frequency contained in the signal.

The sampling frequency value can be chosen as $3-5$ $f_{\max }$. Thus, the sampling frequency can be taken as $4096 \mathrm{~Hz}$. The vibration signal is obtained from the acceleration sensor mounted on the bearing housing when the rotational frequency of the shaft is steady. Because the roller bearing usually turns at a constant speed, the starting and stopping processes are not considered. The inner-race and outer-race defects were created by cutting slots having a width and a depth of $0.15 \mathrm{~mm}$ and $0.13 \mathrm{~mm}$, respectively, with a laser. The roller bearings with the three conditions (normal, inner-race fault (IR fault), and outer-race fault (OR fault)) were tested, and 60 vibration signals from the bearings in each condition were obtained, from which 45 groups were selected at random as the training data.

First, after the original vibration signals were decomposed into ISCs using LCD, the first five ISCs that include the most dominant fault information were chosen and arranged from high to low, according to the frequency components, as $I_{1}(t), I_{2}(t), \ldots, I_{5}(t)$. Then, the fault feature vector $T^{\prime}$ was obtained according to (22), (23), (24), and (25). The ACROA-SVM was used to identify the various patterns. The identification results for the test samples based on LCD preprocessing are shown in Table 6.

In order to make a fair comparison, the same original vibration signals were chosen. These signals were decomposed into IMFs using EMD. Five IMFs were chosen, and they were arranged from high to low, according to the frequency components, as $c_{1}(t), c_{2}(t), \ldots, c_{5}(t)$, and then the fault feature vector $T^{\prime}$ was obtained according to (21), (22), (23), and (24). Next, the ACROA-SVM was used to identify the various patterns. The identification results of the test samples based on EMD preprocessing are shown in Table 7 and are compared with those using LCD.

When used as the preprocessor to extract the energy in each frequency band as ACROA-SVM input vector, both methods (LCD and EMD based) are capable of identifying the faults in the bearings. As mentioned in Section 5, outerrace condition is separated by SVM1 and inner-race condition is separated by SVM2. For SVM1, LCD-ACROA-SVM1 
TABLE 6: The identification results based on LCD and ACROA-SVM method.

\begin{tabular}{|c|c|c|c|c|c|c|c|c|}
\hline Test samples & $E 1$ & $E 2$ & $E 3$ & $E 4$ & E5 & LCD-ACROA-SVM1 & LCD-ACROA-SVM2 & Identification results \\
\hline OR fault & 0.9745 & 0.2185 & 0.0455 & 0.0182 & 0.0028 & $(+1)$ & & OR fault \\
\hline OR fault & 0.9755 & 0.2785 & 0.0515 & 0.0192 & 0.0035 & $(+1)$ & & OR fault \\
\hline OR fault & 0.9735 & 0.2271 & 0.0238 & 0.0118 & 0.0061 & $(+1)$ & & OR fault \\
\hline OR fault & 0.9787 & 0.2010 & 0.0405 & 0.0092 & 0.0069 & $(+1)$ & & OR fault \\
\hline OR fault & 0.9373 & 0.3484 & 0.2070 & 0.0766 & 0.0291 & $(+1)$ & & OR fault \\
\hline IR fault & 0.9846 & 0.1645 & 0.0536 & 0.0213 & 0.0158 & $(-1)$ & $(+1)$ & IR fault \\
\hline IR fault & 0.9766 & 0.1638 & 0.0662 & 0.0210 & 0.0159 & $(-1)$ & $(+1)$ & IR fault \\
\hline IR fault & 0.9718 & 0.1049 & 0.0400 & 0.0304 & 0.0258 & $(-1)$ & $(+1)$ & IR fault \\
\hline IR fault & 0.9950 & 0.0917 & 0.0358 & 0.0120 & 0.0138 & $(-1)$ & $(+1)$ & IR fault \\
\hline IR fault & 0.9961 & 0.0774 & 0.0331 & 0.0220 & 0.0162 & $(-1)$ & $(+1)$ & IR fault \\
\hline Normal & 0.9685 & 0.2077 & 0.1042 & 0.0649 & 0.0611 & $(-1)$ & $(-1)$ & Normal \\
\hline Normal & 0.9621 & 0.2326 & 0.1123 & 0.0715 & 0.0512 & $(-1)$ & $(-1)$ & Normal \\
\hline Normal & 0.9741 & 0.1978 & 0.0908 & 0.0547 & 0.0277 & $(-1)$ & $(-1)$ & Normal \\
\hline Normal & 0.9666 & 0.2137 & 0.1236 & 0.0616 & 0.0312 & $(-1)$ & $(-1)$ & Normal \\
\hline Normal & 0.9764 & 0.2317 & 0.1326 & 0.0636 & 0.0317 & $(-1)$ & $(-1)$ & Normal \\
\hline
\end{tabular}

TABLE 7: The identification results of LCD-ACROA-SVM compared with EMD-ACROA-SVM or LCD-SVM with EMD-SVM.

\begin{tabular}{|c|c|c|c|c|c|c|}
\hline Method & Training samples & Test samples & $C$ & $\sigma$ & Cost time (s) & Test error (\%) \\
\hline LCD-ACROA-SVM1 & 45 & 15 & 287.80 & 2.94 & 5.59 & $\mathbf{0}$ \\
\hline LCD- SVM1 & 45 & 15 & 200 & 1 & / & 6.67 \\
\hline EMD-ACROA-SVM1 & 45 & 15 & 1955.47 & 2.92 & 6.67 & 6.67 \\
\hline EMD-SVM1 & 45 & 15 & 200 & 1 & l & 13.34 \\
\hline LCD-ACROA-SVM2 & 30 & 10 & 285.61 & 4.71 & 4.57 & 0 \\
\hline LCD- SVM2 & 30 & 10 & 200 & 1 & I & 10 \\
\hline EMD-ACROA-SVM2 & 30 & 10 & 1570.16 & 0.99 & 5.74 & 0 \\
\hline EMD-SVM2 & 30 & 10 & 200 & 1 & 1 & 10 \\
\hline
\end{tabular}

classifier has higher accuracy than the LCD-SVM1, the EMDACROA-SVM1, and the EMD-SVM1 classifier. For SVM2, the accuracy rate of the LCD-ACROA-SVM2 and the EMDACROA-SVM2 are the same. In summary, it can be observed from Table 7 that the ACROA-SVM method based on LCD has higher accuracy (i.e., less error) than the method based on EMD and has shorter computation times.

\section{Conclusion}

A roller bearing fault diagnosis method based on LCD energy entropy and the ACROA-SVM was investigated in this paper. First, the original vibration signals were preprocessed using the LCD method. The LCD energy entropy was used as the input to the ACROA-SVM classifier. A theoretical analysis and experimental results show that the ACROASVM combined with the LCD method has higher accuracy and shorter computation times than when combined with EMD. Furthermore, the analysis shows that the LCD method is a self-adaptive signal processing method and is superior to the EMD method. This signal-processing method is adaptive and suitable for nonlinear and nonstationary process.

\section{Conflict of Interests}

The authors declare that there is no conflict of interests regarding the publication of this paper.

\section{Acknowledgments}

The authors would like to acknowledge the financial support from Chinese National Science Foundation Grant (nos. 51075131 and 51175158) and the Nature Science Foundation of Hunan Province, China (no. 11IJ2026).

\section{References}

[1] D. Ho and R. B. Randall, "Optimization of bearing diagnostic techniques using simulated and actual bearing fault signals," Mechanical Systems and Signal Processing, vol. 14, no. 5, pp. 763788, 2000.

[2] Z. Peng, F. Chu, and Y. He, "Vibration signal analysis and feature extraction based on reassigned wavelet scalogram," Journal of Sound and Vibration, vol. 253, no. 5, pp. 1087-1100, 2003.

[3] C. J. Li and S. M. Wu, "On-line detection of localized defects in bearings by pattern recognition analysis," Journal of engineering for industry, vol. 111, no. 4, pp. 331-336, 1989. 
[4] N. E. Huang, Z. Shen, S. R. Long et al., "The empirical mode decomposition and the Hilbert spectrum for nonlinear and non-stationary time series analysis," Proceedings of the Royal Society A, vol. 454, no. 1971, pp. 903-995, 1998.

[5] N. E. Huang, Z. Shen, and S. R. Long, "A new view of nonlinear water waves: the Hilbert spectrum," Annual Review of Fluid Mechanics, vol. 31, pp. 417-457, 1999.

[6] Y. Yang, D. Yu, and J. Cheng, "A fault diagnosis approach for roller bearing based on IMF envelope spectrum and SVM," Measurement, vol. 40, no. 9-10, pp. 943-950, 2007.

[7] J. S. Smith, “The local mean decomposition and its application to EEG perception data," Journal of the Royal Society Interface, vol. 2, no. 5, pp. 443-454, 2005.

[8] G. Rilling and P. Flandrin, "One or two frequencies? The empirical mode decomposition answers," IEEE Transactions on Signal Processing, vol. 56, pp. 85-95, 2008.

[9] Z. Wu and N. E. Huang, "A study of the characteristics of white noise using the empirical mode decomposition method," Proceedings of the Royal Society A, vol. 460, no. 2046, pp. 15971611, 2004.

[10] J. Cheng, D. Yu, and Y. Yang, "Application of support vector regression machines to the processing of end effects of HilbertHuang transform," Mechanical Systems and Signal Processing, vol. 21, no. 3, pp. 1197-1211, 2007.

[11] M. Dätig and T. Schlurmann, "Performance and limitations of the Hilbert-Huang transformation (HHT) with an application to irregular water waves," Ocean Engineering, vol. 31, no. 14-15, pp. 1783-1834, 2004.

[12] S.-s. Long, T.-b. Zhang, and F. Long, "Causes and solutions of overshoot and undershoot and end swing in Hilbert-Huang transform," Acta Seismologica Sinica, vol. 18, no. 5, pp. 602-610, 2005.

[13] M.-K. Hsu, J.-C. Sheu, and C. Hsue, "Overcoming the negative frequencies: instantaneous frequency and amplitude estimation using Osculating Circle method," Journal of Marine Science and Technology, vol. 19, no. 5, pp. 514-521, 2011.

[14] Y. Kopsinis and S. McLaughlin, "Enhanced empirical mode decomposition using a novel sifting-based interpolation points detection," in Proceedings of the 14th WorkShoP on Statistical Signal Processing (SSP '07), pp. 725-729, August 2007.

[15] J. Cheng, Jinde Zheng, and Y. Yang, "Empirical envelope demodulation approarch based on local characteristic-scale decomposition and its applications to mechanical fault diagnosis," Chinese Journal of Mechanical Engineering 10, vol. 46, pp. 148-155, 2010.

[16] M. Zacksenhouse, S. Braun, M. Feldman, and M. Sidahmed, "Toward helicopter gearbox diagnostics from a small number of examples," Mechanical Systems and Signal Processing, vol. 14, no. 4, pp. 523-543, 2000.

[17] V. N. Vapnik, Statistical Learning Theory, John Wiley and Sons, New York, NY, USA, 1998.

[18] C.-L. Jia, H.-S. Wu, and D.-W. Gong, "Coal demand prediction based on a support vector machine model," Journal of China University of Mining and Technology, vol. 36, no. 1, pp. 107-110, 2007.

[19] J. Jang, K. R. Park, J. Kim, and Y. Lee, "New focus assessment method for iris recognition systems," Pattern Recognition Letters, vol. 29, no. 13, pp. 1759-1767, 2008.

[20] K. W. Lau and Q. H. Wu, "Local prediction of non-linear time series using support vector regression," Pattern Recognition, vol. 41, no. 5, pp. 1556-1564, 2008.
[21] B. Long, S. Tian, Q. Miao, and M. Pecht, "Research on features for diagnostics of filtered analog circuits based on LS-SVM," in Proceedings of the AUTOTESTCON, pp. 360-366, September 2011.

[22] M. Saimurugan, K. I. Ramachandran, V. Sugumaran, and N. R. Sakthivel, "Multi component fault diagnosis of rotational mechanical system based on decision tree and support vector machine," Expert Systems with Applications, vol. 38, no. 4, pp. 3819-3826, 2011.

[23] S. Abbasion, A. Rafsanjani, A. Farshidianfar, and N. Irani, "Rolling element bearings multi-fault classification based on the wavelet denoising and support vector machine," Mechanical Systems and Signal Processing, vol. 21, no. 7, pp. 2933-2945, 2007.

[24] K. C. Gryllias and I. A. Antoniadis, "A Support Vector Machine approach based on physical model training for rolling element bearing fault detection in industrial environments," Engineering Applications of Artificial Intelligence, vol. 25, no. 2, pp. 326-344, 2012.

[25] J. Cheng, D. Yu, J. Tang, and Y. Yang, "Application of SVM and SVD technique based on EMD to the fault diagnosis of the rotating machinery," Shock and Vibration, vol. 16, no. 1, pp. 8998, 2009.

[26] M.-J. Han, J.-H. Hsu, K.-T. Song, and F.-Y. Chang, "A new information fusion method for bimodal robotic emotion recognition," Journal of Computers, vol. 3, no. 7, pp. 39-47, 2008.

[27] S. Ari, K. Hembram, and G. Saha, "Detection of cardiac abnormality from PCG signal using LMS based least square SVM classifier," Expert Systems with Applications, vol. 37, no. 12, pp. 8019-8026, 2010.

[28] J. Manikandan and B. Venkataramani, "Design of a real time automatic speech recognition system using Modified One Against All SVM classifier," Microprocessors and Microsystems, vol. 35, no. 6, pp. 568-578, 2011.

[29] S. Bouhouche, L. Laksir Yazid, S. Hocine, and J. Bast, "Evaluation using online support-vector-machines and fuzzy reasoning: application to condition monitoring of speeds rolling process," Control Engineering Practice, vol. 18, no. 9, pp. 10601068, 2010.

[30] C.-J. Lu, T.-S. Lee, and C.-C. Chiu, "Financial time series forecasting using independent component analysis and support vector regression," Decision Support Systems, vol. 47, no. 2, pp. 115-125, 2009.

[31] K. Duan, S. S. Keerthi, and A. N. Poo, "Evaluation of simple performance measures for tuning SVM hyperparameters," Neurocomputing, vol. 51, pp. 41-59, 2003.

[32] F. Imbault and K. Lebart, "A stochastic optimization approach for parameter tuning of Support Vector Machines," in Proceedings of the 17th International Conference on Pattern Recognition (ICPR '04), pp. 597-600, August 2004.

[33] F. Friedrichs and C. Igel, "Evolutionary tuning of multiple SVM parameters," Neurocomputing, vol. 64, no. 1-4, pp. 107-117, 2005.

[34] S. Abe, Advances in Pattern Recognition, Springer, London, UK, 2005.

[35] S. S. Keerthi, "Efficient tuning of SVM hyperparameters using radius/margin bound and iterative algorithms," IEEE Transactions on Neural Networks, vol. 13, no. 5, pp. 1225-1229, 2002.

[36] O. Chapelle, V. Vapnik, O. Bousquet, and S. Mukherjee, "Choosing multiple parameters for support vector machines," Machine Learning, vol. 46, no. 1-3, pp. 131-159, 2002.

[37] X. L. Zhang, X. F. Chen, and Z. J. He, "An ACO-based algorithm for parameter optimization of support vector machines," Expert Systems with Applications, vol. 37, no. 9, pp. 6618-6628, 2010. 
[38] M. J. Abdi, S. M. Hosseini, and M. Rezghi, "A novel weighted support vector machine based on particle swarm optimization for gene selection and tumor classification," Computational and Mathematical Methods in Medicine, vol. 2012, Article ID 320698, 7 pages, 2012.

[39] F. Ardjani, K. Sadouni, and M. Benyettou, "Optimization of SVM multiclass by particle swarm (PSO-SVM)," in Proceedings of the 2nd International Workshop on Database Technology and Applications (DBTA '10), November 2010.

[40] K. Chandramouli and E. Izquierdo, "Image classification using chaotic particle swarm optimization," in Proceedings of the International Conference on Image Processing (ICIP '06), pp. 3001-3004, October 2006.

[41] L. Yu, H. Chen, S. Wang, and K. K. Lai, "Evolving least squares support vector machines for stock market trend mining," IEEE Transactions on Evolutionary Computation, vol. 13, no. 1, pp. 87102, 2009.

[42] T.-J. Hsieh and W.-C. Yeh, "Knowledge discovery employing grid scheme least squares support vector machines based on orthogonal design bee colony algorithm," IEEE Transactions on Systems, Man, and Cybernetics B, vol. 41, no. 5, pp. 1198-1212, 2011.

[43] B. Alatas, "ACROA: artificial chemical reaction optimization algorithm for global optimization," Expert Systems with Applications, vol. 38, no. 10, pp. 13170-13180, 2011.

[44] B. Alatas, "A novel chemistry based metaheuristic optimization method for mining of classification rules," Expert Systems with Applications, vol. 39, pp. 11080-11088, 2012.

[45] P. K. Nag, Engineering Thermodynamics, McGraw-Hill, 4th edition, 2008.

[46] D. Huang, "Practical implementation of the Hilbert-Huang transform algorimth," Acta Oceanologica Sinica, vol. 25, no. 1, pp. 1-11, 2003.

[47] Y. Yu, Y. YuDejie, and C. Junsheng, "A roller bearing fault diagnosis method based on EMD energy entropy and ANN," Journal of Sound and Vibration, vol. 294, no. 1-2, pp. 269-277, 2006.

[48] J. G. Proakis and D. G. Manolakis, Digital Signal Processing: Principles, Algorithms and Applications, 2nd edition, 1992. 

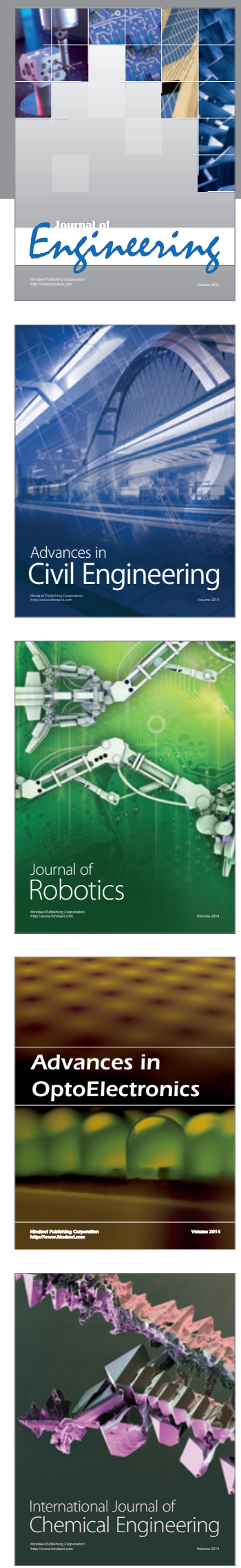

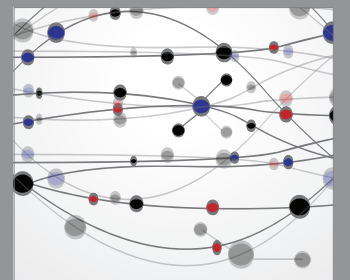

The Scientific World Journal
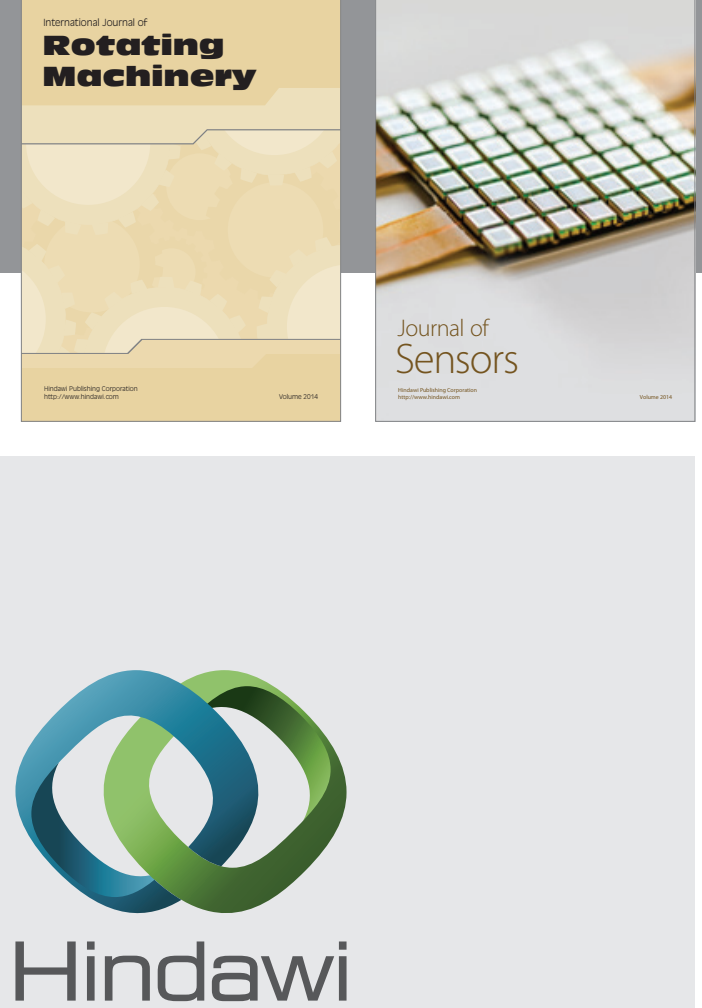

Submit your manuscripts at http://www.hindawi.com
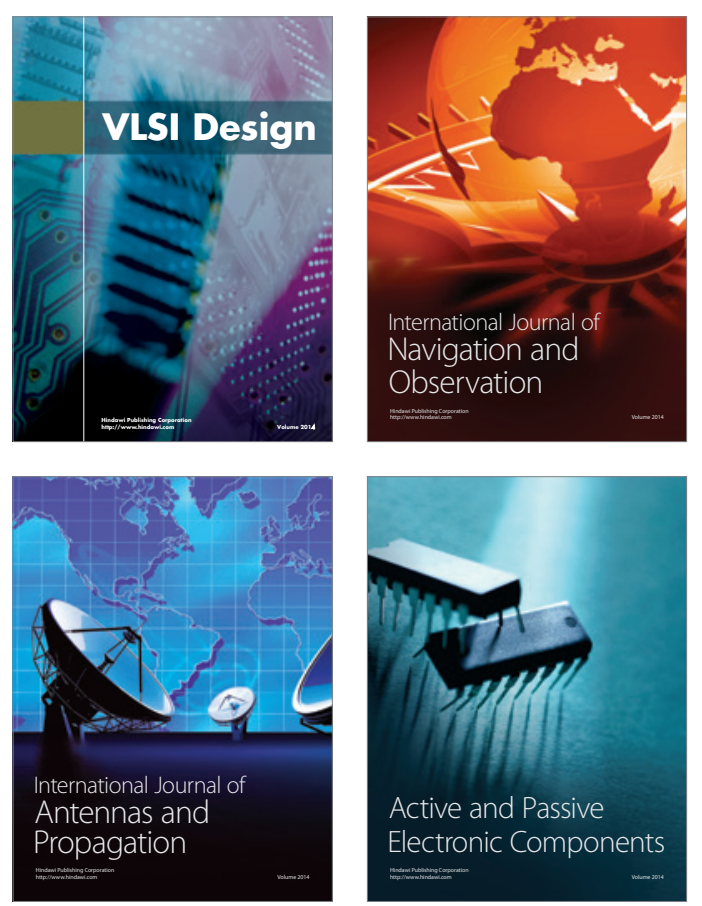
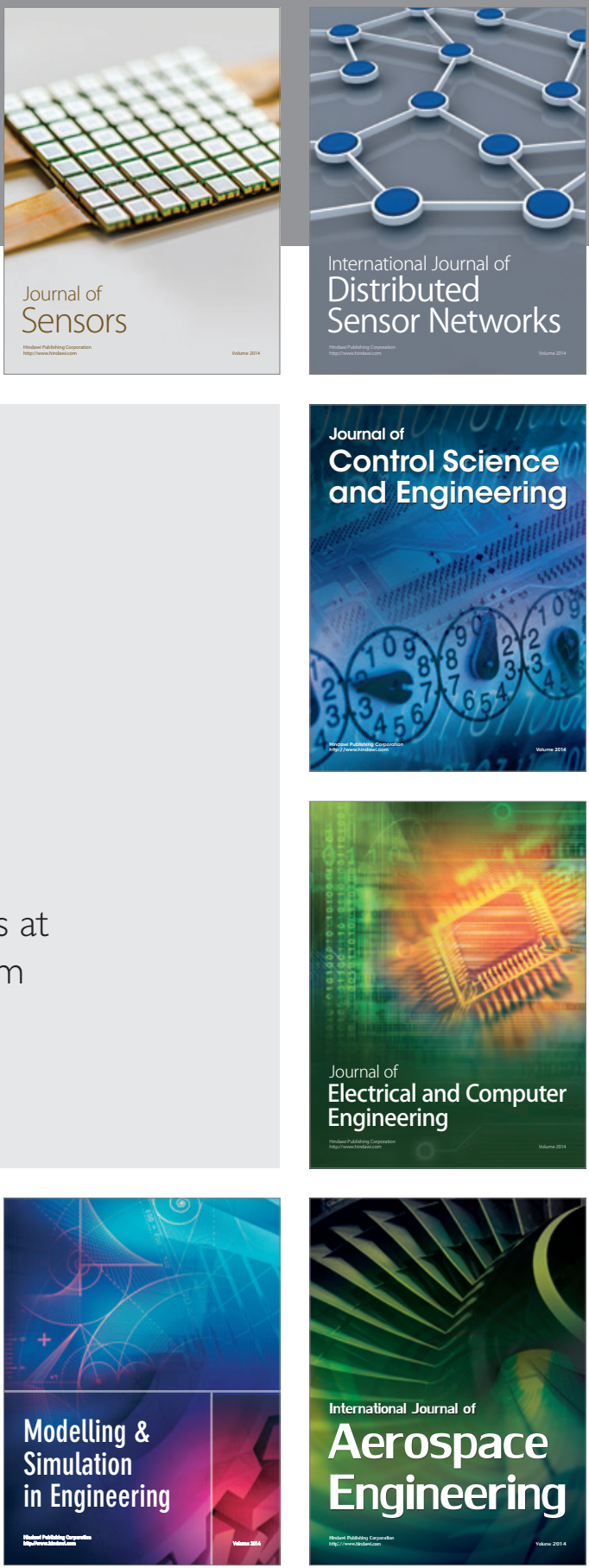

Journal of

Control Science

and Engineering
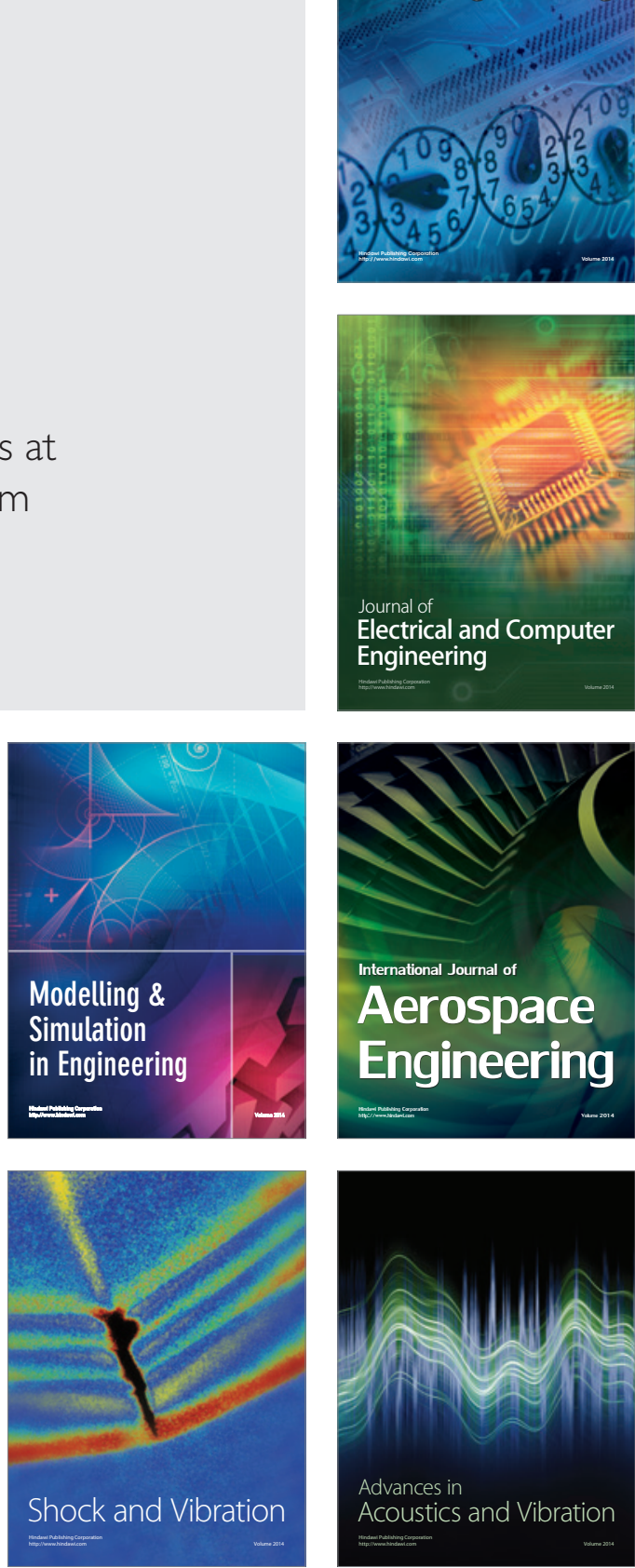